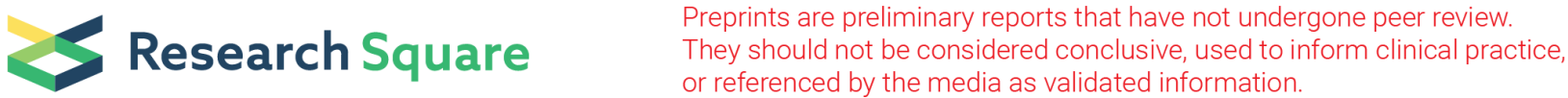

\section{Genomic analysis reveals fifty years of antimicrobial resistance evolution in husbandry Escherichia coli from China}

\author{
Lu Yang \\ China Agricultural University \\ Yingbo Shen \\ Chinese Academy of Sciences \\ Junyao Jiang \\ China Agricultural University

\section{Xueyang Wang} \\ China Agricultural University \\ Dongyan Shao \\ China Agricultural University \\ Margaret Lam \\ Monash University \\ Kathryn Holt \\ Monash University \\ Bing Shao \\ China Agricultural University \\ Congming Wu \\ China Agricultural University \\ Jianzhong Shen \\ China Agricultural University \\ Timothy Walsh \\ University of Oxford

\section{Stefan Schwarz} \\ Freie Universität Berlin
}

\section{Yang Wang ( $\sim$ wangyang@cau.edu.cn )}

China Agricultural University https://orcid.org/0000-0002-5928-9377

\section{Zhangqi Shen}

China Agricultural University

\section{Article}

Keywords: Escherichia coli, antimicrobial resistance, meat production, antimicrobial agents

Posted Date: May 19th, 2021

DOI: https://doi.org/10.21203/rs.3.rs-484138/v1

License: @ (i) This work is licensed under a Creative Commons Attribution 4.0 International License. Read Full License 
Version of Record: A version of this preprint was published at Nature Food on March 24th, 2022. See the published version at https://doi.org/10.1038/s43016-022-00470-6. 


\section{Abstract}

Antimicrobial agents have been used in meat production for decades and its consumption is considered an key driver for the emergence and dissemination of antimicrobial resistance (AMR). However, large-scale studies on AMR changes in animal isolates since the introduction of antimicrobial usage remain scarce. We applied whole genome sequencing analysis to 982 animal-derived Escherichia coli collected in China from 1970s to 2019 and found increasing trends for the presence of numerous antimicrobial resistance genes (ARGs), including those conferring resistance to critically important agents for veterinary (florfenicol and norfloxacin) and human medicine (colistin, cephalosporins, and meropenem). Extensive diversity and increasing complexity of ARGs and their associated mobile genetic elements (MGEs) such as plasmids were also observed. The plasmids, IncC, IncHI2, IncK, Incl, IncX and IncF played a key role as highly effective vehicles for disseminating ARGs. Correlation analysis also revealed an association between antimicrobial production and emergence of ARGs at a spatial and temporal level. Prohibiting or strictly curtailing antimicrobial use in animals will potentially negate the current trends of AMR as the bacterial genome is highly changeable and using different drugs of the same class, or even unrelated classes, may co-select for MGEs carrying a plethora of co-existing ARGs. Therefore, limiting or ceasing antimicrobial use in animals to control AMR requires careful consideration.

\section{Introduction}

Antimicrobial resistance (AMR) has become a global health and development threat. Currently, at least 700,000 people die annually of infections caused by antimicrobial-resistant organisms, and this number is projected to rise to at least 10 million with accumlated costs of approx. $\$ 100$ trillion by $2050^{1}$. This relentless increase in AMR is exacerbated by global antibiotic consumption (AMC), which increased by nearly $70 \%$ during the 2000 s with much of the AMC occuring in animalproduction $^{2}$. In the USA, for example, over $70 \%$ (by weight) of antimicrobial agents assigned to classes that are medically important in human medicine, such as $\beta$-lactams, tetracyclines, macrolides and colistin, are sold for use in animals ${ }^{3}$. Such extensive AMC has driven the rapid emergence of multidrug-resistant (MDR) pathogens in both humans ${ }^{4}$ and animals ${ }^{5}$. Whilst horizontal gene transfer via plasmids and transposons may account for inter-bacterial dissemination ${ }^{6}$, the interconnections between animals and humans either through the food chain, direct contact or environmental routes, also contribute to the widespread of $\mathrm{AMR}^{7}$.

Monitoring longitudinal AMR data is not only essential for analysing trends, but also for the early detection of emerging resistance traits i.e. globally coordinated surveillance studies have been conducted since the $1990 \mathrm{~s}^{8}$, of which Escherichia coli, a commensal bacterium, is considered as an excellent AMR indicator ${ }^{9}$. Moreover, $E$. coli is the most frequently reported clinical pathogen worldwide ${ }^{10}$. In 2015, WHO launched the Global Antimicrobial Resistance Surveillance System (GLASS) to facilitate AMR surveillance in bacteria causing critical infections and to assess AMR trends across WHO regions ${ }^{11}$. However, no comparable framework exists for AMR surveillance in animals, and few country-specific monitoring or surveillance studies have been conducted in Europe ${ }^{12}$ and the United States ${ }^{9}$. In low-middle countries (LMIC)s, the OIE are liasing with the Fleming Fund to leverage such data but is restricted to 24 countries ${ }^{13,14}$. These programs show large AMR variations across countries, which may be due to a myriad of reasons including technical e.g. antimicrobial susceptibility testing (AST) methods. One of the earliest (2000-2018), large LMIC AMR studies on bacteria including E. coli from animals revealed an AMR increase among pig and chicken isolates ${ }^{15}$. AMR surveillance of $E$. coli from infirm chickens in China between 1993 and 2013 showed an increasing trend in MDR ${ }^{16}$. Most AMR surveillance studies on bacteria from animals are limited in their spatial and temporal sampling, and mostly focus on phenotypic data only. Moreover, an association between AMR and animal AMC has seldom been investigated.

In China, antimicrobial agents have been used as feed additives in the animal farming industry since the $1970 \mathrm{~s}^{17}$. The annual per capita consumption of meat has increased from $10.3 \mathrm{~kg}$ in 1978 to $57.6 \mathrm{~kg}$ in $2019^{18}$, and this dramatic 
increase has resulted in a correspondingly rapid increase in animal AMC; thereby, enflaming the AMR problem. However, despite these mitigating factors, changes in animal AMR levels remain poorly understood. Herein, we investigated the AMR trends of animal-derived E. coli in China from 1970s to 2010s. The evolution and transmission of ARGs alongside the plasticity of ARG-carrying plasmids were also evaluated, focusing on specific AMR markers to clinically important antimicrobial agents used in animals ${ }^{19}$ and humans ${ }^{20}$.

\section{Results}

\section{Dynamics of Escherichia coli populations from animals in China}

Altogether, 982 isolates (450 from our laboratory, 532 from the NCBI database as of December 2019) were derived from animals or their breeding environments in 26 Chinese provinces (Fig. 1, Table S1). Most were collected from South and East China ( $n=572)$, where the meat production industry is mainly concentrated ${ }^{21}$. Isolates were collected from chickens $(n=567)$ and pigs $(n=258)$, sheep $(n=62)$, cattle $(n=46)$, ducks $(n=4)$, goose $(n=1)$, and also 44 E. coli isolates from flies within poultry-farm environments. All isolates were chronologically divided into four groups: 1970s-1980s $(n=113$ isolates), 1990s ( $n=33$ isolates), 2000s ( $n=169$ isolates), and 2010 s ( $n=667$ isolates).

The phylogenetic tree of the $982 \mathrm{E}$. coli isolates showed substantial diversity within the population and was clustered into 9 lineages using Bayesian model-based population structures (BAPS) analysis (L1-L9, Fig. 1a). All lineages, except L2 and $L 5$, comprised isolates from all temporal groups. The eight common phylogenetic groups $(A, B 1, B 2, C, D, E, F$, clade I), classified based on the Clermont $E$. coli phylo-typing method ${ }^{22}$, comprised $42.97 \%, 37.07 \%, 1.63 \%, 5.60 \%, 4.89 \%, 3.05 \%$, $4.68 \%$ and $0.10 \%$ of the population, respectively (Table S1). Groups A $(n=422)$ and B1 $(n=364)$ accounted for $80.0 \%$ of the population. The representation of isolates from phylogroup $\mathrm{C}$ declined over time from $22.1 \%$ in the $1970-80 \mathrm{~s}, 15.2 \%$ in the 1990 s, $4.7 \%$ in the 2000 s and $2.6 \%$ in the 2010 s. Isolates from 1990 s included more group B2 (15.15\%) and group F (27.3\%), which would more likely to be extraintestinal pathogenic E. coli (ExPEC) $)^{23}$.

Analysis of the two clades (C1, n=31 and C2, n=32), with proportion of $62.5 \%$ and $38.7 \%$, respectively, from $1970 \mathrm{~s}-1980 \mathrm{~s}$

samples, showed that the ARGs (bla $a_{\mathrm{CTX}-\mathrm{M}}, b / a_{\mathrm{OXA}}, b / a_{\mathrm{NDM}}$, oqxAB, qnrS, $m c r$, and $f l o R$ ) and the plasmid replicons (IncHI2, Incl2, IncX1, IncX3, and IncB/O/K/Z) existed primarily in isolates from the later time groups in both $\mathrm{C} 1$ and $\mathrm{C} 2$ clades (mean prevalence of these ARG and plasmid were $0 \%$ and $3 \%$ in the 1970 s- 1990 s vs $80 \%$ and $70 \%$ in the 2000 s- 2010 s, see Fig. S1b).

\section{MLST diversity and patterns}

MLST of 982 isolates demonstrated significant diversity (Fig. S2), with isolates assigned to 226 distinct sequence types (STs), except for 67 isolates with novel STs. More than half of the STs $(54.0 \%, n=122)$ were represented by a single isolate. ST10 was the most populated and contained 79 isolates (8.0\%) across all four chronological datasets. Other major STs included ST101 ( $n=38,3.9 \%)$, ST156 ( $n=36,3.7 \%)$, ST48 ( $n=36,3.7 \%)$, ST155 ( $n=28,2.9 \%)$, ST224 ( $n=22,2.2 \%)$, ST46 $(n=20,2.0 \%)$ and ST88 $(n=20,2.0 \%)$. The diversity of STs was higher in the 2010 s data set, although this may reflect the larger number of isolates in this data set. The prevalence of ST48 increased in the years following 2010 (Fig. S3). Conversely, ST88, ST5086, ST351, ST90, ST345, ST1, ST100 and ST56 were more prevalent before 2000, while ST156, ST354, ST224, ST101 and ST46 were mainly observed after 2000 (Fig. S3). STs that were more prevalent in the most recent groups generally had relatively higher ARG presence (Fig. S4) e.g. ST156 and ST354 isolates had a mean ARG count of 23.9, which was significant higher than the overall mean of $15.0(p<0.01$, Table S1).

\section{Pan-genome analysis}

Pangenomic analysis of the 982 E. coli isolates revealed an open pangenome of 66,767 genes and showed no evidence of reaching a plateau (Fig. S5a), indicating that genes were expanded as the sample size increased. Contigs were classified 
as most likely originating from chromosomes or plasmids according to the prediction results from mlplasmids ${ }^{24}$. The chromosomal core genome was predicted to contain 1,724 genes using a 99\% threshold, and 57,434 accessory genes were identified with mean 2,837 genes per genome (Table S1). The isolates from the first three chronological groups (1970s2000s inclusive) were found to be more conserved based for their chromosomal pangenome accumulation curves (Fig. S5b), while group 4 (2010s) appeared to have a more open pangenome, which may be account for the larger spatial and host representation. The accessory gene counts per genome varied over time, ranging from 2,311 to 3731 . Isolates collected in the 1970s-1980s and 1990s had more chromosomal accessory genes (mean=2223), compared to those from 2000s and 2010s (mean=2152, $p<0.001$ ), whilst the plasmid accessory genes increased from mean 193 in isolates from 1970s-1980s to mean 292 in isolates from 2010s ( $<$ 0.001, Table S1).

Plasmid replicon marker analysis also indicates a slight increase in the number of plasmids over time with a mean count of 10.0 in isolates collected from the 1970s-1980s to 12.5 in isolates from 2010 ( $p<0.001$, Fig. S6a). The prevalence of several ARG-associated plasmids, such as IncX3, IncX4, Incl2 and IncHI2, were significantly higher in 2010s than in the 1970s-1980s (Fig. S8). The IncX3, Incl2 and IncB/O/K/Z plasmids were rarely detected between 1970s and 1990s (0\%-6.06\%), but increasingly presented in isolates from the 2010 s (14.54\% - 31.78\%, Fig. S8). The plasmid profile was generally similar across isolates from different sectors. However, comparing isolates from pigs, the isolates from chickens had a significant higher prevalence of IncC (8.99\% vs. $2.33 \%)$, Inc B/O/K/Z (14.46\% vs $2.33 \%)$, IncK (14.46\% vs. $2.33 \%)$, Incl1 (25.04\% vs. $15.50 \%)$ and IncHI2 (20.99\% vs. $13.95 \%)$, and a significant lower prevalence of IncX1 (16.75\% vs. $23.64 \%)$, IncX3 (8.82\% vs. $14.73 \%)$, IncX4 (2.29\% vs. $7.36 \%)$ and IncY (14.11\% vs. $22.87 \%)$ type plasmids.

\section{Temporal changes of AMR, ARG and virulence gene (VG) profiles}

Antimicrobial susceptibility testing of $450 \mathrm{E}$. coli isolates and ARG screening of $982 \mathrm{E}$. coli genomes revealed an increasing trend in AMR across the near-50 years time span (Fig. 2 a-c). We observed an increase in resistance to more recently approved and/or less commonly used veterinary drugs $(p<0.01)$, such as florfenicol, apramycin, norfloxacin, and ceftiofur, and the drugs exclusively used in human medicine, such as aztreonam and meropenem (Fig. 2a). Similar trends were observed in their corresponding ARGs in the full genome set (Fig. 2a and c). In contrast, resistance to antimicrobial agents commonly employed since the 1970's, e.g. tetracycline, trimethoprim/sulfamethoxazole and chloramphenicol, remained relatively high throughout the 1970s-2010s (48.67\%-79.78\%, Fig. 2a, Table S2). Correspondingly, the presence of ARGs also remained high in the full geneome set (Fig. 2c). Porcine isolates had a relatively higher prevalence of ARGs conferring resistance to colistin and fluoroquinolones $(65.5 \%$ vs. $44.9 \%$ in other hosts, $p<0.001)$, whilst sheep isolates had the lowest prevalence of ARGs (mean count of 5.97 vs 15.57 in others, $p<0.001$, Fig. 2c).

We specifically examined the resistance to $\beta$-lactams and phenicols which are key antimicrobial agents for both human and/or veterinary medicine. The rates of resistance to penicillins (ampicillin) and penicillins $+\beta$-lactamase inhibitors (amoxicillin/clavulanic acid), remained low in the 1970 s-1980s (24.39\% and $13.41 \%$, respectively), but rose rapidly since 1990 s $(67.86 \%$ and $71.43 \%$, respectively) and remain at a high-level (72.86\% and $53.77 \%$, respectively, in 2010 s).

Resistance to cephalosporins and aztreonam have increased over time (from $1.22 \%$ and $1.22 \%$ to $52.24 \%$ and $34.17 \%$ ) and resistance to meropenem was most noticeable in the 2010 s (1.01\%, Fig. 2a, Table S2). The average counts of ESBL genes per genome for each chronological group in the full genome set were $0.24,0.73,0.83$ and 1.16, respectively (Fig. 3a); $70.37 \%$ of the isolates $(n=691)$ were found to contain at least one ESBL gene and the percentage in each time group increased from $22.12 \%$ in 1970 s-1980s to $69.70 \%-77.66 \%$ in 1990 s-2010s. Among the ESBL-positive isolates, $28.08 \%$ were also positive for bla NDM $(n=194)$. Although the use of chloramphenicol in food-producing animals was banned in China in

$2002^{25}$, florfenicol, a fluorinated thiamphenicol derivative, is still in use. The prevalence of chloramphenicol resistance has remained relatively high with a slight decline in the 2000 s $(60.71 \%-43.26 \%$, Fig. $2 a)$ and the most prevalent chloramphenicol resistance genes have changed from catA1 to catB3, $\mathrm{cm} / A$ and $f l o R$ (which mediates bacterial resistance to both chloramphenicol and florfenicol) (Fig. 2c, Table S3). Genetic context analysis of phenicol resistance genes indicate 
that catA is linked with only a few ORFs encoding hypothetical proteins and likely associated with an IncP plasmid, while $c a t B, c m / A$ and $f l o R$ genes were found to be associated with IncHI2 plasmids along with other ARGs (Figs. 5 and 6 ).

Four fluoroquinolones (norfloxacin, pefloxacin, lomefloxacin, and ofloxacin) were banned from usage in food-producing animals by $2016^{26}$, and colistin was prohibited for use as an animal growth promoter in April $2017^{27}$. We therefore used these two time points to further group isolates in order to investigate how these restrictions may have affected AMR and ARG profiles. Resistance to norfloxacin increased rapidly from the 1990s to the 2000s before declining in the 2010s (Fig. S11a). Correspondingly, the average counts of PMQR genes for each chronoloigical group were $0.02,0.18,0.45$ and 0.73 , respectively (Table S3). The prevalence of oqxAB and qnrS increased; qnrB and qepA continue to remain low, with a slight decrease in $\operatorname{aac}\left(6^{\prime}\right)-\operatorname{lb}-\operatorname{cr}(19.39 \%, 102 / 526$ before 2016 vs. 17.02\%, 24/141, after 2016). In contrast, the prevalence of colistin resistance and the gene, $m c r-1$, increased rapidly during the 2000s (Fig. S11b), but decreased from $44.27 \%$ $(n=278 / 628)$ in 2010-2016 to $23.08 \%(n=9 / 39)$ in 2017-2019, after the ban of colistin as growth promoter was enforced.

For virulence genes profiles, most isolates were detected to be hypovirulent, with 769 VGs (Table S3) functionally clustering into 16 classes and 100 groups (Fig. S9). No obvious trends or changes in VGs were observed. Pig isolates had a lower prevalence of virulence genes associated with adherence, autotransporters, invasion and iron uptake compared to isolates from chickens. Sheep isolates horbored highest virulence genes but lowest number of ARGs (Fig. 3a).

\section{Correlation analysis of the gross output value of veterinary medicine for ARGs and VGs}

To understand the association between AMC and AMR, we used the antimicrobial production data as an alternative measure to veterinary drug usage, which was unavailable in China prior to 2018. In general, the gross output value of the veterinary drugs produced in China during the different chronological periods (Table S5) were positively correlated with the detected average ARG counts ( $r_{\text {Pearson }}=0.95, p=0.004$; Fig. $4 a$ ), inversely correlated with the average virulence gene count $\left(r_{\text {Pearson }}=-0.86, p=0.028\right.$; Fig. $\left.4 \mathrm{c}\right)$, and exhibited no correlation with the average plasmid-associated replicon count

$\left(r_{\text {Pearson }}=0.18, p=0.733\right.$; Fig. $\left.4 b\right)$. We observed no correlation between ARGs and VGs $(r$ Pearson $=-0.04, p=0.243)$ and moderate positive association between plasmid replicon number and ARGs ( $r$ Pearson $=0.35, p<0.001$ ), and virulence genes (rPearson = 0.30, p < 0.001; Fig. S12).

\section{The transfer of ARGs}

We applied the DISCOVER method commonly used for testing the somatic alterations in cancer ${ }^{28}$ as a statistical approach for analyzing the co-occurrence of ARGs and plasmid replicons to infer the transmission of resistance genes. Several plasmid replicons were associated with ARGs (Fig. 5), such as IncC with the ESBL-related gene bla $a_{\mathrm{CMY}}$, Incl2 with $m c r$, IncX3 with the carbapenem resistance gene $b / a_{\mathrm{NDM}}$, IncHI2 with seven resistance gene classes, among others. The cooccurrence of ARGs and plasmid replicons largely remained stable when the analysis was restricted to different chronological-groups, with the IncC plasmid replicon being the one exception which was associated with the trimethoprim resistance gene dfrA23 in the 1970s-1990s, but then associated with the ESBL gene bla CMY $_{\text {after }} 2000$ (Fig. S13). ARG genetic context analysis was used to investigated clustering of ARGs in assembled contigs, and showed that isolates from the 1970s-1990s possessed smaller clusters with fewer links between ARGs, but those from the 2000 and 2010 group gradually possessed more densely populated ARG regions (e.g. the $\beta$-lactamase genes or aminoglycoside resistance genes, Fig. 6 and S14). For example, we found that catB and cm/A co-existed with the bla $a_{0 X A-1}, b / a_{0 X A-10}, b / a_{N D M}, a p h\left(3^{\prime}\right)$ $X V$, ant(2")-la, aadA, ant(3")-la, the PMQR gene $\operatorname{aac}\left(6^{\prime}\right)-l b-c r$, and the rifampicin resistance gene arr (Fig. 6a-c). Additionally, oqxAB gene was the first to be detected in category of PMQR during the 1970s-1990s, while the appearance of $\operatorname{acc}\left(6^{\prime}\right)-I b-c r$ in the 2000s was the only PMQR that co-existed with other ARGs such as bla OXA-1 $_{1}$ and arr. The mcr-1 gene was always associated with the IS 30 family transposase, ISAp/1, but the flanking regions have diversified over time.

\section{Discussion}

Page 6/20 
Previous studies investigating AMR in animals in China have been limited at both a spatial and/or temporal level ${ }^{29-33}$, while our study presents a nation-wide surveillance report encompassing isolates from different food-producing animals from the 1970s, a time when antimicrobial agents seldom used in veterinary medicine and have since largely replaced traditional Chinese herbs ${ }^{34}$, to 2019 . Worldwide, China is now one of the largest users of veterinary antimicrobial agents ${ }^{21}$. In this study, we observed a significant association between veterinary antimicrobial production and the enrichment of ARGs, further demonstrating that the long-term use of antimicrobials in animals is one of the key drivers of AMR. As AMC in animals increased over the five decades, so the evolution of E. coli from animal origins with expanded AMR profiles continue to expand via antimicrobial selection.

To address this critical issue, the Chinese government announced a series of directives aimed at reducing AMR in animals. Concurrently, several programs were launched for AMR monitoring ${ }^{35,36}$ with several antimicrobial classes banned (chloramphenicol in 2002, four fluoroquinolones in 2016) and colistin prohibited as growth promoter in April 2017 25-27. The overall prevalence of AMR profiles and corresponding ARGs; however, did not decline in the 2010s, but exhibited divergent trends (Fig. 3). The observation, that the dissemination of colistin resistance in $E$. coli dramatically reduced after the withdrawal of colistin, was consistent with our previous study ${ }^{37}$. This reduction may have been mediated by the following: 1. the $m c$-mediated target modification on lipid A moiety of LPS is the sole transferable mechanism of colistin resistance in $E$. coli ${ }^{38}$. 2. $m c r-1$ expression can impose a considerable fitness cost for $E$. coli carrying $m c r$-bearing plasmids ${ }^{39-42}$. 3 . The ban of colistin as a growth promoter significantly reduced the selection pressure for $m c r-1$ which would have previously occurred. 4 . The observation that $m c r$ genes are seldom found together with other ARGs on the same mobile genetic elements (Fig. 6) reduces the likelihood for co-selection by other antimicrobial agents.

In contrast, the ban of chloramphenicol and the four fluoroquinolones from use in animals did not result in a phenotypic AMR decrease or a reduction in their corresponding ARGs. This might be attributable to the following: 1 . Other antimicrobial agents within the same class, including florfenicol (phenicols) as well as ciprofloxacin, danofloxacin, enrofloxacin, and sarafloxacin (fluoroquinolones) were still used in animals and may have provided selection pressure for particular genotypes. 2. The co-selection by other antimicrobial agents might have contributed to the persistence and continual spread of resistance to phenicols and fluoroquinolones e.g. the PMQR gene $\operatorname{acc}\left(6^{\prime}\right)-I b-c r$ is frequently found to be located in a class 1 integron with $c a t B, b / a_{0 X A}$ and $a r r{ }^{43,44}$. Co-occurrence of these genes was observed in 64.1\% (91/142) of our isolates that were positive for $\operatorname{acc}\left(6^{\prime}\right)-l b-c r$. 3 . The immediate genetic environment have become increasingly complex due to the accumulation of additional ARGs via mobile genetic elements (MGEs) (Fig. 6). These genes are likely to be mobilised together and the exposure to any one of these correspsonding antimicrobial agents could result in the coselection of the entire gene cluster or plasmid. 4. Multiple mobile resistance genes mediating diverse resistance mechanisms relating to chloramphenicol resistance (floR-related efflux pump and cat-associated enzymatic inactivation, $c f$-mediated target site modification) and fluoroquinolone resistance (oqxAB and qepA-related efflux pump, acc( $\left.6^{\prime}\right)-I b-c r-$ related enzymatic inactivation and qnr-associated target protection) render the loss of these resistance traits more difficult than a single resistance mechanism e.g. mcr-1. 5. Fluoroquinolone resistance is more frequently mediated by mutations in specific chromosomal target genes, such as gyrA, gyrB, parC or parE. Such chromosomal mutations are very stable, especially under continuous selection pressure imposed by the use of fluoroquinolones in animals. Finally, fluoroquinolones can further induce mutations causing resistance to other classes of antimicrobials ${ }^{45}$, which continue to strengthen their competitive positions.

Another difficulty hindering AMR control is the prosperity of horizontal gene transfer by MGEs. Acquisition of ARGs is greatly aided by a variety of MGEs, among which plasmids play a crticial role ${ }^{46}$. To date, 27 major plasmid incompatibility groups have been associated with ARGs in Enterobacteriaceae ${ }^{47,48}$. The co-occurrence of ARGs and several plasmid replicon types was commonly detected within our isolates (Fig. 5). The Incl2, IncX3, IncHI2, IncC and IncF types were linked with ARGs, with some of these associations highlighted in previous studies ${ }^{49}$. The IncF, IncC and IncX plasmid types were 
recently identified to be the most prevalent types in carbapenem-resistant $E$. coli ${ }^{50}$, while Incl2, IncHI2 and IncX4 types are commonly linked with $m c r-1$ transmission ${ }^{51}$. As the increasing prevalence of ARGs was consistent with increasing detection of these plasmid replicons (Fig. 2c, S2b, S8), any AMR containment necessitates the mitigation of these multiresistance plasmids and therefore strategies to inhibit their transmission should be further explored.

We acknowledge several limitations in this study. A few isolates $(n=60)$ were collected several decades ago and their collection details (e.g., exact location) were no longer available. Apart from those downloaded from NCBI, all remaining isolates from the 1990s ( $n=28,84.85 \%$ ) were collected from a chicken farm in Jiangsu Province, and may represent a sampling bias. Additional sampling bias may also result from the available data from online database given the skew towards sequencing isolates with specific ARGs. However, given the extensive sources involved in this study, and the obvious huge diversity of these genomes, the bias is likely to be minor.

Stricter use of antimicrobial agents in animals is a strating to become a global trend; at least in theroy. As such, the European Union banned the use of antimicrobial growth promoters by 2006 (Regulation (EC) No. 1831/2003) and more recently, the Ministry of Agriculture and Rural Affairs, People's Republic of China has launched a regulation (No. 194) to stop growth-promoting medicines as feed additives after $1^{\text {st }}$ July $2020^{52}$. It should be noted that antimicrobial agents are still irreplaceable for the treatment and prevention of infectious diseases caused by bacteria. It should also be noted that only healthy livestock animals provide high quality meat products for human consumption which is an increasing global trend. Thus, sick animals need an adequate treatment, which in the case of bacterial infections also involves the appopriate application of antimicrobial agents. However, fortified with the understanding that antimicrobial agents will select specific resistant genotypes, it is crticial to design AMR reduction stratgeies that considers the co-existence and cotransfer of ARGs i.e., interventions exploring separate classes of antimicrobials for humans and animals.

\section{Materials And Methods}

\section{Sample collection and whole genome sequencing}

A total of 450 E. coli isolates from animals in China collected since the 1970 s were collated from our previous studies. Among which, 110 isolates were provided by the China Institute of Veterinary Drug Control ${ }^{53}$, including 82 isolates collected from pig, cattle, sheep etc. in 1970s-1980s and 28 isolates collected from chicken in 1990s. The remaining 340 isolates were collected from our previous AMR epidemiological investigations on different regions ${ }^{54-56}$, which includes 84 from chicken in 2002-2006, 191 from chikcen in 2008-2013, and 65 from pig in 2008-2014. All of these isolates were collected with no preference. All isolates were recovered by identification on EMB medium (Land Bridge, Beijing, China) followed by MALDI-TOF MS (Matrix-Assisted Laser Desorption/lonization Time of Flight Mass Spectrometry). Of the 450 isolates, 169 have been sequenced and partly published (BioProject Accession number: PRJNA417344). Genomic DNA from the remaining 281 isolates was extracted for whole genome sequencing using the Wizard Genomic DNA Purification Kit (Promega, Beijing, China) following the manufacturer's instructions. DNA libraries were constructed using KAPA HyperPrep Kit Illumina platforms (Roche, Basel, Weitzerland) following standard protocols, and then sequenced on Illumina HiSeq X Ten platform (Annoroad, Beijing, China). Raw sequence data of clinical strains were assembled using SPAdes ${ }^{57}$ v3.13.1 via the Unicycler ${ }^{58}$ v0.4.7 assembly pipeline. Assemblies were deposited in NCBI (BioProject Accession number: PRJNA718052). Three isolates were selected for long-read sequencing, because they were collected from the early temporal group or were assigned to the clades in which we were particularly interested. MinION library preparation was performed using the Rapid Barcoding Sequencing kit (SQK-RBK004) according to the the manufacturer's (Oxford Nanopore, Oxford, UK) standard protocol. The constructed library was loaded into the Flow Cell R9.4 (FLO-MIN106) on a MinION device and run with the SQK-RBK004_plus_Basecaller script in MinKNOW1.5.12 software. De-multiplexing and basecalling were conducted using guppy 3.2.4. The long-read sequencing data was combined with their corresponding short-read Illumina data and used to generate de novo hybrid assemblies with Unicycler ${ }^{58}$ v0.4.7. Additional genomes were 
downloaded from the National Center for Biotechnology Information (NCBI) Pathogen detection database

(https://www.ncbi.nlm.nih.gov/pathogens/isolates\#/search/). There were $n=1728$ isolates retrieved with the search criteria "species_taxid:562" and "geo_loc_name: China*" (E. coli from China) on December $9^{\text {th }} 2019$. Among which, 606 isolates collected from food-producing animals (chicken, gallus gallus, pig, swine, duck, ovis aries, bos taurus, et al.) were secected. In addition to 74 genomes originating from our lab, 532 E. coli genomes collected between 1976-2019 were downloaded. The details of these projects involved in this study were listed in Table S6. About half of these isolates $(n=243)$ were collected with no specific selection criteria, 5 isolates were submitted as virulent isolates, 5 isolates were related to specific phage, and 279 isolates were involved in AMR research. However, some of these resistant isolates were sequenced by our previous study and the other non-resistant isolates in the same epidemiological investigations have also been added. The large sample size of this collective dataset may account for a potential sampling bias to a certain extent.

\section{Antimicrobial susceptibility testing}

The minimum inhibitory concentrations (MICs) of the $450 \mathrm{E}$. coli isolates were determined using the agar diffusion method according to Clinical and Laboratory Standards Institute (CLSI) document M100, $30^{\text {th }}$ ed. ${ }^{59}$ and The European Committee on Antimicrobial Susceptibility Testing (EUCAST) standard $v 10.0^{60}$. Altogether, 16 antimicrobial agents from nine classes were selected based on their usage in the animal industry and/or in human medicine, and included gentamicin, apramycin, tetracycline, tigecycline, norfloxacin, florfenicol, chloramphenicol, ampicillin, amoxicillin/clavulanic acid, meropenem, cefazolin, cefoxitin, ceftiofur, trimethoprim/sulfamethoxazole, aztreonam and colistin.

\section{Phylogenetic analysis}

The full set of 982 genomes were used to generate a core-genome SNP alignment and construct a phylogenetic tree, using Parsnp v1.1.2 in the Harvest package ${ }^{61}$. The mid-point rooted phylogenetic tree was annotated in ITOL (https://itol.embl.de/). The Bayesian model-based population structures were identified with HierBAPS ${ }^{62}$ v6.0. Following initial phylogenetic analysis, two clades, $\mathrm{C} 1$ and $\mathrm{C} 2$, were selected for further analysis. A representative isolate from each clade was selected for long-read sequencing as detailed above, yielding high-quality complete chromosome sequences for use as reference genomes. Illumina data sets were aligned against the chromosomes of high-quality complete assemblies using Bowtie $2^{63}$. Single nucleotide polymorphisms (SNPs) were identified, it for all of the sequenced genomes with SAMtools ${ }^{64}$ v1.3.1 using RedDog v1beta.11 (https://github.com/katholt/RedDog) pipeline with default settings. For each of the two clades, core SNPs (conserved in $>95 \%$ genomes) were used for subsequent phylogenetic analysis. Gubbins ${ }^{65}$ v2.3.4 was used to identify and filter out putative chromosomal recombination imports within each lineage, before generating recombination-free maximum likelihood phylogenetic trees using FastTree ${ }^{66}$ v2.1.10. The minimum spanning tree of MLST was generated in BioNumerics v7.6.

\section{The gene content analysis}

Illumina read sets were screened against the $E$. coli 7-locus multi-locus sequence type (MLST) database ${ }^{67}$, ARGannot $^{68}$ database, PlasmidFinder ${ }^{69}$ database and virulence factor database (VFDB, http://www.mgc.ac.cn/VFs/) using SRST2 ${ }^{70}$ v0.2.0 to assign sequence types (STs) and detect acquired AMR genes, plasmid replicons and VGs, respectively, in each genome. All the VGs were grouped according to the information from VFDB. The results were transformed into a binary table in $\mathrm{R}^{71} \mathrm{v} 3.6 .0$ to analyse the presence/absence of acquired AMR gene alleles and prevalence of each gene in different isolates grouped by temporal or host information. Contigs in each genome assembly were classified as chromosomal or plasmid sequences using mlplasmids ${ }^{24}$ v1.0.0 the posterior probability cut-off at 0.5 . The resulting contig sets (chromosomal vs. plasmid) were each annotated with Prokka ${ }^{72}$ v1.11 and subjected to a pan-genome analysis with Roary ${ }^{71}$ v3.6.0, separately. The resulting gene content matrix comprised 982 genomes and was used as input for plotting in $\mathrm{R}$ v3.6.3. Gene networks showing the linkage of genes within contigs were generated in $\mathrm{Gephi}^{73}$. 


\section{Statistical analyses}

Statistical analyses were conducted with R v3.6.3. The Pearson correlation analysis was used to determine the correlation between the gross output value of veterinary medicine and gene content, and the pairwise correlation between ARGs, VGs, and plasmid replicons. Differences between the numbers of ARGs/VGs/plasmid replicons per isolate in different decadespecific dataset (1970s-1980s, 1990s, 2000s, 2010s) were assessed by the Wilcoxon test. Gene prevalence differences between each decade-specific dataset were assessed with the $\chi 2$ test $(n \geq 50)$ or Fisher's exact test $(n<50)$.

We used the R package Discover (Discrete Independence Statistic Controlling for Observations with Varying Event Rates) v0.9.3, a novel statistical independence test, to identify the pariwise co-occurrence and mutual exclusivity of ARGs and plasmid replicons in the genomes ${ }^{28}$. This package assesses pairwise co-occurrence and mutual exclusivity by counting how many genomes have both of the two genes and comparing this to the number of genmoes expected to have such an overlap by chance if these genes were appeared independent. In addition, mutual exclusion can be detected, and the detection of mutual exclusion would be improved. In short, Discover identifies more significant co-occurrences and mutual exclusivities without increasing the false positive rate.

\section{Declarations}

\section{Acknowledgements}

This work was supported in part by grants from the National Natural Science Foundation of China (81861138051, 81991535 and 31761133004 ) and the UK Medical Research Council (project DETER-XDR-China-HUB, grant number $\mathrm{MR} / \mathrm{S} 013768 / 1)$.

\section{References}

1 O'Neill, J. Antimicrobial Resistance: Tackling a crisis for the health and wealth of nations. (2014).

2 Van Boeckel, T. P. et al. Global antibiotic consumption 2000 to 2010: an analysis of national pharmaceutical sales data. Lancet Infect Dis 14, 742-750, doi:10.1016/S1473-3099(14)70780-7 (2014).

3 O'Neill, J. Tackling drug-resistant infections globally: Final report and recommendations. 2016. HM Government and Welcome Trust: UK (2018).

$4 \quad$ O'Neill, J. Antimicrobials in agriculture and the environment: reducing unnecessary use and waste. The review on antimicrobial resistance, 1-44 (2015).

5 Aarestrup, F. M., Kruse, H., Tast, E., Hammerum, A. M. \& Jensen, L. B. Associations between the use of antimicrobial agents for growth promotion and the occurrence of resistance among Enterococcus faecium from broilers and pigs in Denmark, Finland, and Norway. Microbial Drug Resistance 6, 63 (2000).

6 Carattoli, A. Plasmids and the spread of resistance. International Journal of Medical Microbiology 303, 298-304, doi:10.1016/j.jmm.2013.02.001 (2013).

7 Hernando-Amado, S., Coque, T. M., Baquero, F. \& Martinez, J. L. Defining and combating antibiotic resistance from One Health and Global Health perspectives. Nature Microbiology 4, 1432-1442, doi:10.1038/s41564-019-0503-9 (2019).

8 Hawser, S. Surveillance programmes and antibiotic resistance: worldwide and regional monitoring of antibiotic resistance trends. Handbook of Experimental Pharmacology, 31-43, doi:10.1007/978-3-642-28951-4_3 (2012). 
9 McEwen, S. A. \& Fedorka-Cray, P. J. Antimicrobial use and resistance in animals. Clinical Infectious Diseases 34 Suppl 3, S93-S106, doi:10.1086/340246 (2002).

10 Global Antimicrobial Resistance and Use Surveillance System (GLASS) Report Early implementation 2020. (2020).

11 Global Antimicrobial Resistance Surveillance System (GLASS) Report Early implementation 2016-2017. (2017).

12 Authority, E. F. S., Prevention, E. C. f. D. \& Control. The European Union Summary Report on Antimicrobial Resistance in zoonotic and indicator bacteria from humans, animals and food in 2017/2018. EFSA Journal 18 (2020).

13 The Fleming Fund, <https://www.flemingfund.org>

14 OIE, <https://www.oie.int>

15 Van Boeckel, T. P. et al. Global trends in antimicrobial resistance in animals in low- and middle-income countries. Science 365, doi:10.1126/science.aaw1944 (2019).

16 Chen, X. et al. Escherichia coli isolates from sick chickens in China: changes in antimicrobial resistance between 1993 and 2013. The Veterinary Journal 202, 112-115, doi:10.1016/j.tvjl.2014.06.016 (2014).

17 Zhao, Y. et al. Feed additives shift gut microbiota and enrich antibiotic resistance in swine gut. Science of The Total Environment 621, 1224-1232 (2018).

$18 \mathrm{Yu}, \mathrm{X}$. Meat consumption in China and its impact on international food security: Status quo, trends, and policies. Journal of Integrative Agriculture, 989-994 (2015).

19 OIE. OIE list of antimicrobial agents of veterinary importance.

https://www.oie.int/fileadmin/Home/eng/Our_scientific_expertise/docs/pdf/AMR/A_OIE_List_antimicrobials_July2019.pdt (2018).

20 WHO. Critically important antimicrobials for human medicine. https://apps.who.int/iris/bitstream/handle/10665/312266/9789241515528-eng.pdf?ua=1 (2019).

21 Van Boeckel, T. P. et al. Global trends in antimicrobial use in food animals. Proceedings of the National Academy of Sciences of the United States of America 112, 5649-5654 (2015).

22 Clermont, O., Christenson, J. K., Denamur, E. \& Gordon, D. M. The Clermont Escherichia coli phylo-typing method revisited: improvement of specificity and detection of new phylo-groups. Environmental Microbiology Reports 5 (2013).

23 Johnson, J. R. \& Russo, T. A. Molecular Epidemiology of Extraintestinal Pathogenic Escherichia coli. Ecosal Plus (2018).

24 Arredondo-Alonso, S. et al. mlplasmids: a user-friendly tool to predict plasmid- and chromosome-derived sequences for single species. Microbial Genomics 4, doi:10.1099/mgen.0.000224 (2018).

25 MOA. Announcement of the Ministry of Agriculture and Rural People's Republic of China No. 193. http://www.moa.gov.cn/ztzl/ncpzxzz/fffg/200709/t20070919_893091.htm (2002).

26 MOA. Announcement of the Ministry of Agriculture and Rural People's Republic of China No. 2292. http://www.moa.gov.cn/nybgb/2015/jiuqi/201712/t20171219_6103873.htm (2015).

27 MOA. Announcement of the Ministry of Agriculture and Rural People's Republic of China No. 2428. http://www.moa.gov.cn/nybgb/2016/dibaqi/201712/t20171219_6102822.htm (2016). 
28 Canisius, S., Martens, J. W. M. \& Wessels, L. F. A. A novel independence test for somatic alterations in cancer shows that biology drives mutual exclusivity but chance explains most co-occurrence. Genome Biology 17, 261 (2016).

29 Jun, Z. L. Changes of Antimicrobial Resistance of E.coli. Chinese Journal of Veterinary Drug (2001).

30 Chao, Y. Analysis of Escherichia coli Antibiotic Resistance in Different Periods. Beijing Agriculture (2011).

31 Lei, L. C., Dan, Z., Han, W. Y., Feng-Tao, J. I. \& Wei-Dong, X. U. Antibiotic Sensitivity Detection and Analysis of Escherichia coli Isolated Clinically in China. Chinese Journal of Veterinary 25 (2005).

32 Meng-Yuan, L. et al. Investigation of Antibiotic Resistance and Predominant Serotypes of Porcine Pathogenic E.coli. Chinese Journal of Veterinary 24 (2004).

33 Zhang, P. et al. Surveillance of Antimicrobial Resistance among Escherichia coli from Chicken and Swine, China, 2008-2015. Veterinary Microbiology 203, 49 (2017).

34 Zhang, K. J. Review of Veterinary Traditional Chinese Medicine and Formulae in the 20th Century. Journal of Chinese Veterinary Medicine, 31-33 (1999).

35 Yuan, z., Zhang, m., Dai, M. \& Huang, I. Analysis of veterinary antimicrobial resistance and Suggestions for prevention and control. Chinese Journal of Veterinary Medicine 46, 7-11 (2012).

36 Tang, K. L. et al. Restricting the use of antibiotics in food-producing animals and its associations with antibiotic resistance in food-producing animals and human beings: a systematic review and meta-analysis. The Lancet Planetary Health 1, e316-e327 (2017).

37 Wang, Y. et al. Changes in colistin resistance and mcr-1 abundance in Escherichia coli of animal and human origins following the ban of colistin-positive additives in China: an epidemiological comparative study. The Lancet Infectious Diseases 20, 1161-1171, doi:https://doi.org/10.1016/S1473-3099(20)30149-3 (2020).

38 Wang, R., Dorp, L. V., Shaw, L. P., Bradley, P. \& Balloux, F. The global distribution and spread of the mobilized colistin resistance gene mcr-1. Nature Communications 9, 1179 (2018).

39 Wu, R. et al. Fitness Advantage of mcr-1-Bearing Incl2 and IncX4 Plasmids in Vitro. Frontiers in Microbiology 9 , doi:10.3389/fmicb.2018.00331 (2018).

40 Ke et al. Fitness cost of a mcr-1-carrying IncHI2 plasmid. PLoS One (2018).

41 Nang, S. C. et al. Fitness cost of mcr-1-mediated polymyxin resistance in Klebsiella pneumoniae. Journal of Antimicrobial Chemotherapy, 6 (2018).

42 Yang, Q. et al. Balancing mcr-1 expression and bacterial survival is a delicate equilibrium between essential cellular defence mechanisms. Nature Communications.

43 Moura, A., Pereira, C., Henriques, I. \& Correia, A. Novel gene cassettes and integrons in antibiotic-resistant bacteria isolated from urban wastewaters. Research in Microbiology 163, 92-100 (2012).

44 Chang, C. Y., Fang, Y. T., Tsai, S. M., Chang, L. L. \& Yu, W. L. Characterization of class 1 integrons and gene cassettes in clinical isolates of Klebsiella pneumoniae from Taiwan. Diagnostic Microbiology \& Infectious Disease 65, 214-216 (2009). 
45 Pribis, J. P. et al. Gamblers: An Antibiotic-Induced Evolvable Cell Subpopulation Differentiated by Reactive-OxygenInduced General Stress Response. Molecular Cell (2019).

46 Bennett, P. M. Plasmid encoded antibiotic resistance: acquisition and transfer of antibiotic resistance genes in bacteria. British Journal of Pharmacolofy 153 Suppl 1, S347-357, doi:10.1038/sj.bjp.0707607 (2008).

47 Carattoli, A. Resistance plasmid families in Enterobacteriaceae. Antimicrobial Agents and Chemotherapy 53, 22272238 (2009).

48 Laura, V., Aurora, G. F., Daniela, F. \& Alessandra, C. Replicon sequence typing of IncF plasmids carrying virulence and resistance determinants. Journal of Antimicrobial Chemotherapy, 2518-2529 (2010).

49 Partridge, S. R., Kwong, S. M., Firth, N. \& Jensen, S. O. Mobile Genetic Elements Associated with Antimicrobial Resistance. Clinical Microbiology Reviews 31, doi:10.1128/CMR.00088-17 (2018).

50 Kopotsa, K., Osei Sekyere, J. \& Mbelle, N. M. Plasmid evolution in carbapenemase-producing Enterobacteriaceae: a review. Annals of New York Academy of Sciences 1457, 61-91, doi:10.1111/nyas.14223 (2019).

51 Matamoros, S. et al. Global phylogenetic analysis of Escherichia coli and plasmids carrying the mcr-1 gene indicates bacterial diversity but plasmid restriction. Scientific Reports 7, 15364, doi:10.1038/s41598-017-15539-7 (2017).

52 MOA. Announcement of the Ministry of Agriculture and Rural People's Republic of China No. 194.

http://www.moa.gov.cn/nybgb/2019/201907/202001/t20200103_6334292.htm (2019).

$53 \mathrm{Li}, \mathrm{S}$. et al. Investigation of integrons/cassettes in antimicrobial-resistant Escherichia coli isolated from food animals in China. Science China 53, 613-619 (2010).

$54 \mathrm{Li}$, L. et al. Characterization of antimicrobial resistance and molecular determinants of beta-lactamase in Escherichia coli isolated from chickens in China during 1970-2007. Veterinary Microbiology 144, 505-510 (2010).

55 Dai, L. et al. Characterization of antimicrobial resistance among Escherichia coli isolates from chickens in China between 2001 and 2006. Fems Microbiology Letters 286, 178-183 (2008).

56 Shen, Z., Wang, Y., Shen, Y., Shen, J. \& Wu, C. Early emergence of mcr-1 in Escherichia coli from food-producing animals. The Lancet Infectious Disease 16, 293, doi:10.1016/S1473-3099(16)00061-X (2016).

57 Bankevich, A. et al. SPAdes: a new genome assembly algorithm and its applications to single-cell sequencing. Journal of Computational Biology 19, 455-477, doi:10.1089/cmb.2012.0021 (2012).

58 Wick, R. R., Judd, L. M., Gorrie, C. L. \& Holt, K. E. Unicycler: Resolving bacterial genome assemblies from short and long sequencing reads. PLoS Computational Biology 13, e1005595, doi:10.1371/journal.pcbi.1005595 (2017).

59 Institute, C. a. L. S. CLSI M100-ED30:2020 Performance Standards for Antimicrobial Susceptibility Testing, 30th Edition. http://em100.edaptivedocs.net/(2020).

60 Testing, E. C. o. A. S. Breakpoint tables for interpretation of MICs and zone diameters, Version 10.0. http://www.eucast.org (2020).

61 Treangen, T. J., Ondov, B. D., Koren, S. \& Phillippy, A. M. The Harvest suite for rapid core-genome alignment and visualization of thousands of intraspecific microbial genomes. Genome Biology 15, 1-15 (2014). 
62 Cheng, L., Connor, T. R., Sirén, J., Aanensen, D. M. \& Corander, J. Hierarchical and Spatially Explicit Clustering of DNA Sequences with BAPS Software. Molecular Biology and Evolution 30 (2013).

63 Langmead, B. \& Salzberg, S. L. Fast gapped-read alignment with Bowtie 2. Nature Methods 9, 357-359, doi:10.1038/nmeth.1923 (2012).

$64 \mathrm{Li}, \mathrm{H}$. et al. The Sequence Alignment/Map format and SAMtools. Bioinformatics 25, 2078-2079, doi:10.1093/bioinformatics/btp352 (2009).

65 Croucher, N. J. et al. Rapid phylogenetic analysis of large samples of recombinant bacterial whole genome sequences using Gubbins. Nucleic Acids Research 43, e15, doi:10.1093/nar/gku1196 (2015).

66 Price et al. FastTree 2-Approximately Maximum-Likelihood Trees for Large Alignments. PLoS One(2010).

67 Maiden, M. et al. Multilocus sequence typing: A portable approach to the identification of clones within populations of pathogenic microorganisms. Proceedings of the National Academy of Sciences (1998).

68 Gupta, S. K. et al. ARG-ANNOT, a new bioinformatic tool to discover antibiotic resistance genes in bacterial genomes. Antimicrobial Agents and Chemotherapy 58 (2014).

69 Carattoli, A. et al. In Silico Detection and Typing of Plasmids using PlasmidFinder and Plasmid Multilocus Sequence Typing. Antimicrobial Agents and Chemotherapy 58, 3895-3903 (2014).

70 Inouye, M. et al. SRST2: Rapid genomic surveillance for public health and hospital microbiology labs. Genome Medicine 6, 90, doi:10.1186/s13073-014-0090-6 (2014).

71 Page, A. J. et al. Roary: rapid large-scale prokaryote pan genome analysis. Bioinformatics 31, 3691-3693, doi:10.1093/bioinformatics/btv421 (2015).

72 Seemann, T. Prokka: rapid prokaryotic genome annotation. Bioinformatics 30, 2068-2069, doi:10.1093/bioinformatics/btu153 (2014).

73 Bastian, M., Heymann, S. \& Jacomy, M. in Proceedings of the Third International Conference on Weblogs and Social Media, ICWSM 2009, San Jose, California, USA, May 17-20, 2009.

\section{Figures}




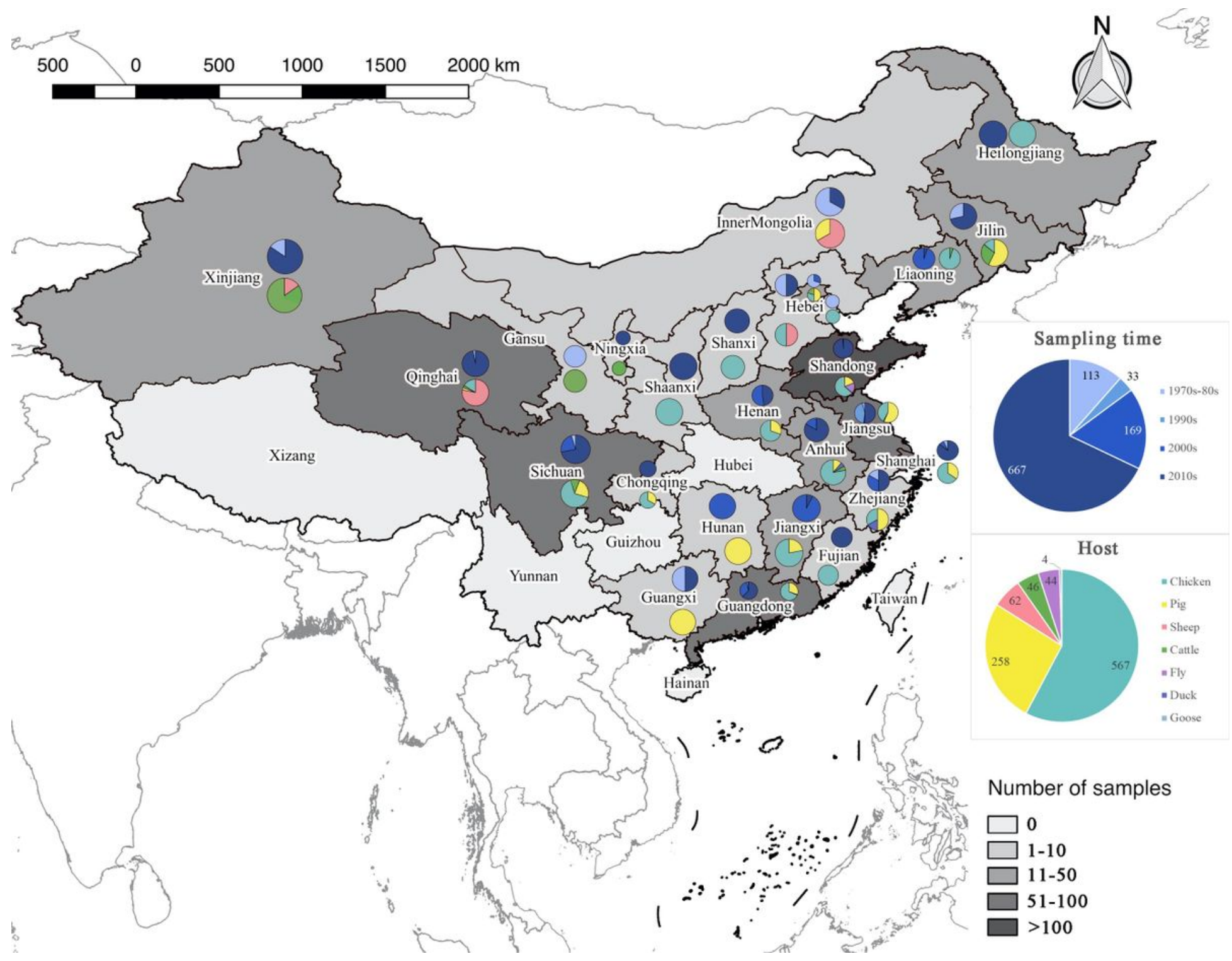

Figure 1

The origins of $982 \mathrm{E}$. coli isolates from 26 Chinese provinces. All isolates were grouped by sampling time and host. The two sets of pie charts show the temporal and host distribution of isolates overall and for each province. Note: The designations employed and the presentation of the material on this map do not imply the expression of any opinion whatsoever on the part of Research Square concerning the legal status of any country, territory, city or area or of its authorities, or concerning the delimitation of its frontiers or boundaries. This map has been provided by the authors. 


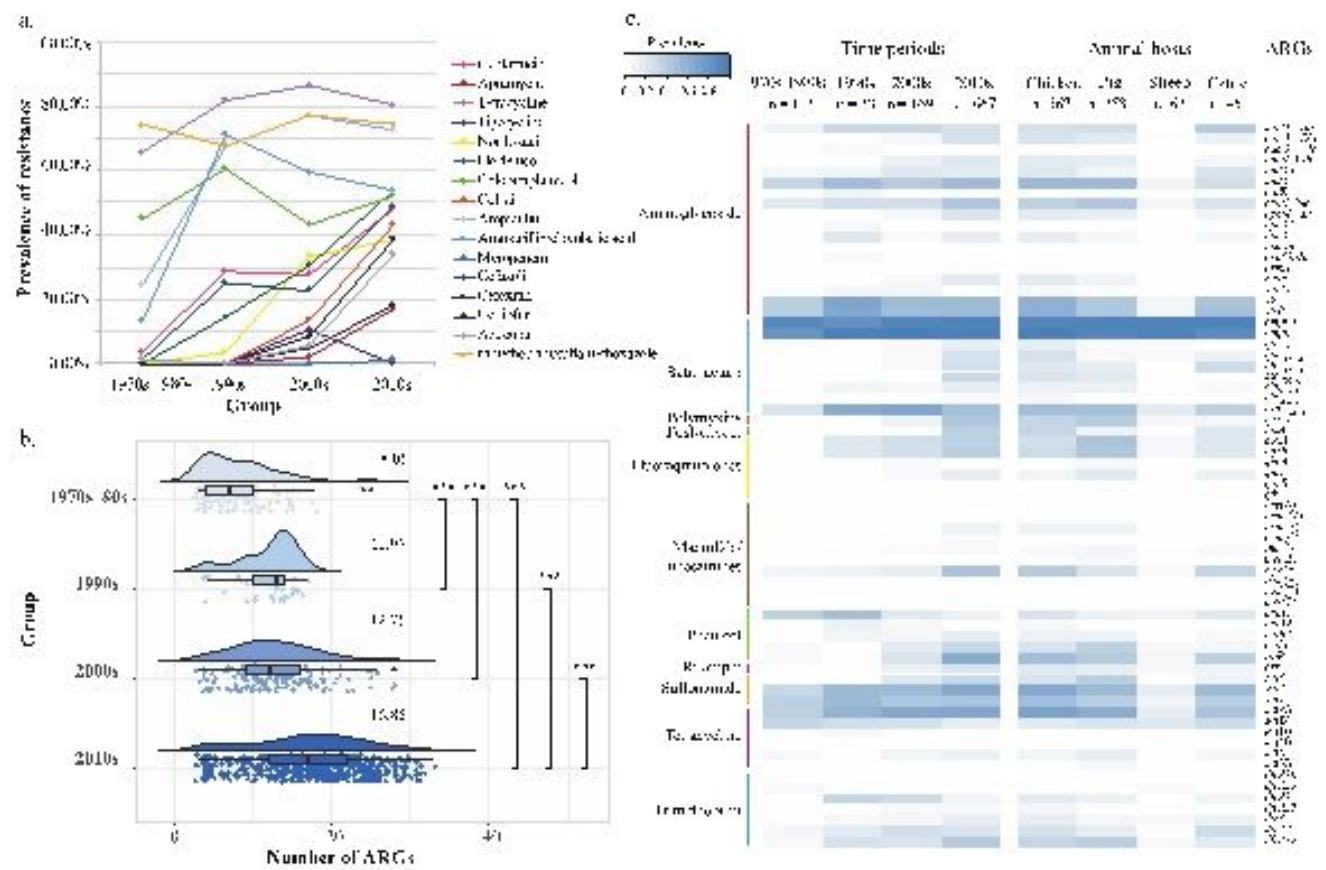

Figure 2

Temporal changes in AMR. a. Temporal changes in AMR phenotypes $(n=450)$. Different colors represent the different antimicrobial agents. The values were presented in Table S2. b. The distribution of numbers of ARGs per genome in different sampling time groups $(n=982)$. The mean ARG count for each group is noted. The "*” on the right represent $p$ values of pairwise groups using Wilcox test. *: $p<0.05$; $* *$ : $p<0.01$; $* * *: p<0.001$. c. ARG prevalence grouped by sampling time and host $(n=982)$. ARGs that were detected at $>5 \%$ prevalence in any one of the sampling groups are shown. They were clustered by their resistance of different groups of antimicrobial agents and colored by antimicrobial classes on the left.
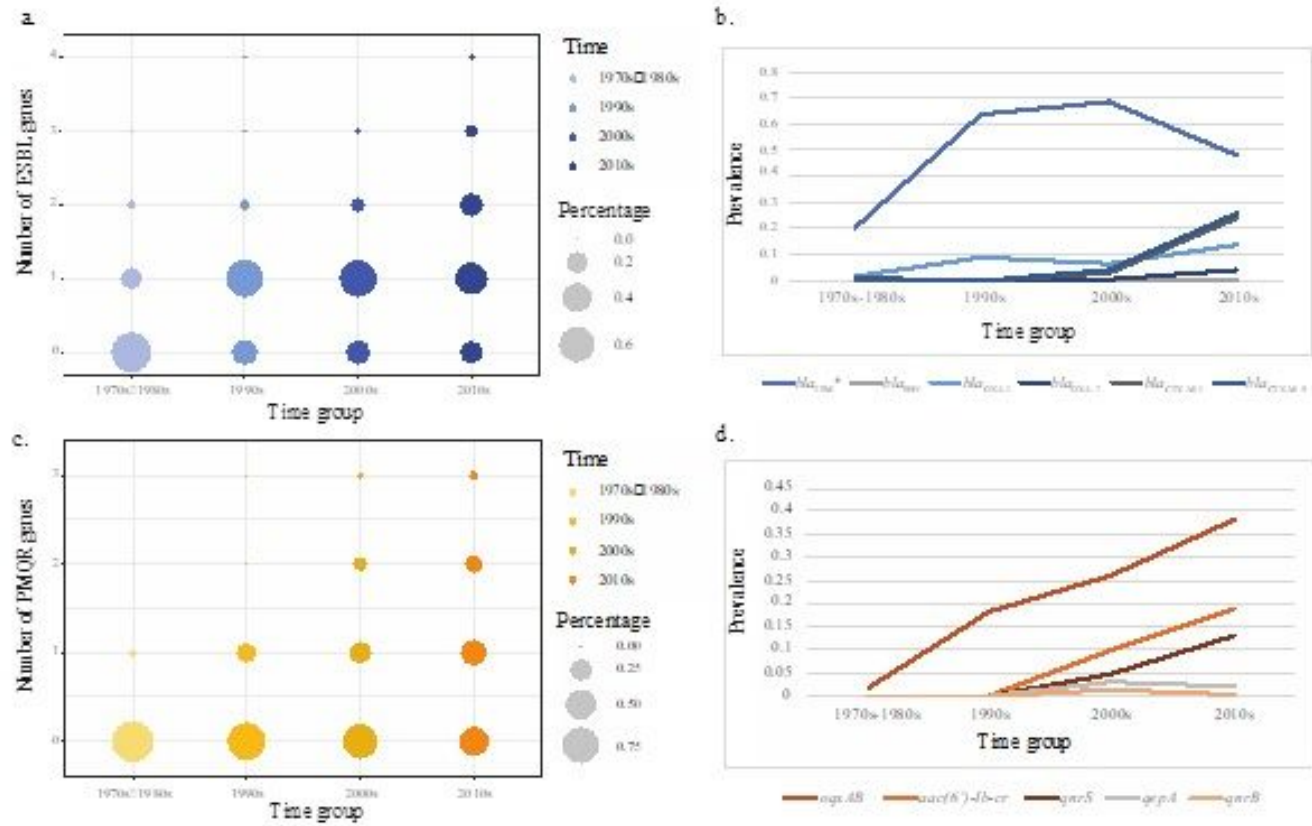

\section{Figure 3}


Temporal changes in ESBL and PMQR gene profiles amongst the full genome set $(n=982)$. a. Number of ESBL genes per isolate over sampling time. b. Prevalence of ESBL genes over sampling time. c. Number of PMQR genes per isolate over sampling time. d. Prevalence of ESBL(b)/PMQR(d) genes over sampling time. The size of each circle in a and $c$ corresponds to the proportion of isolates (percentage). blaTEM*: specify only ESBL producing blaTEM genes.

a.

75 -

$p=0.004$

$\hat{r}_{\text {Pearson }}=0.95$,

$\mathrm{Cl}_{95 \%}[0.59,0.99]$

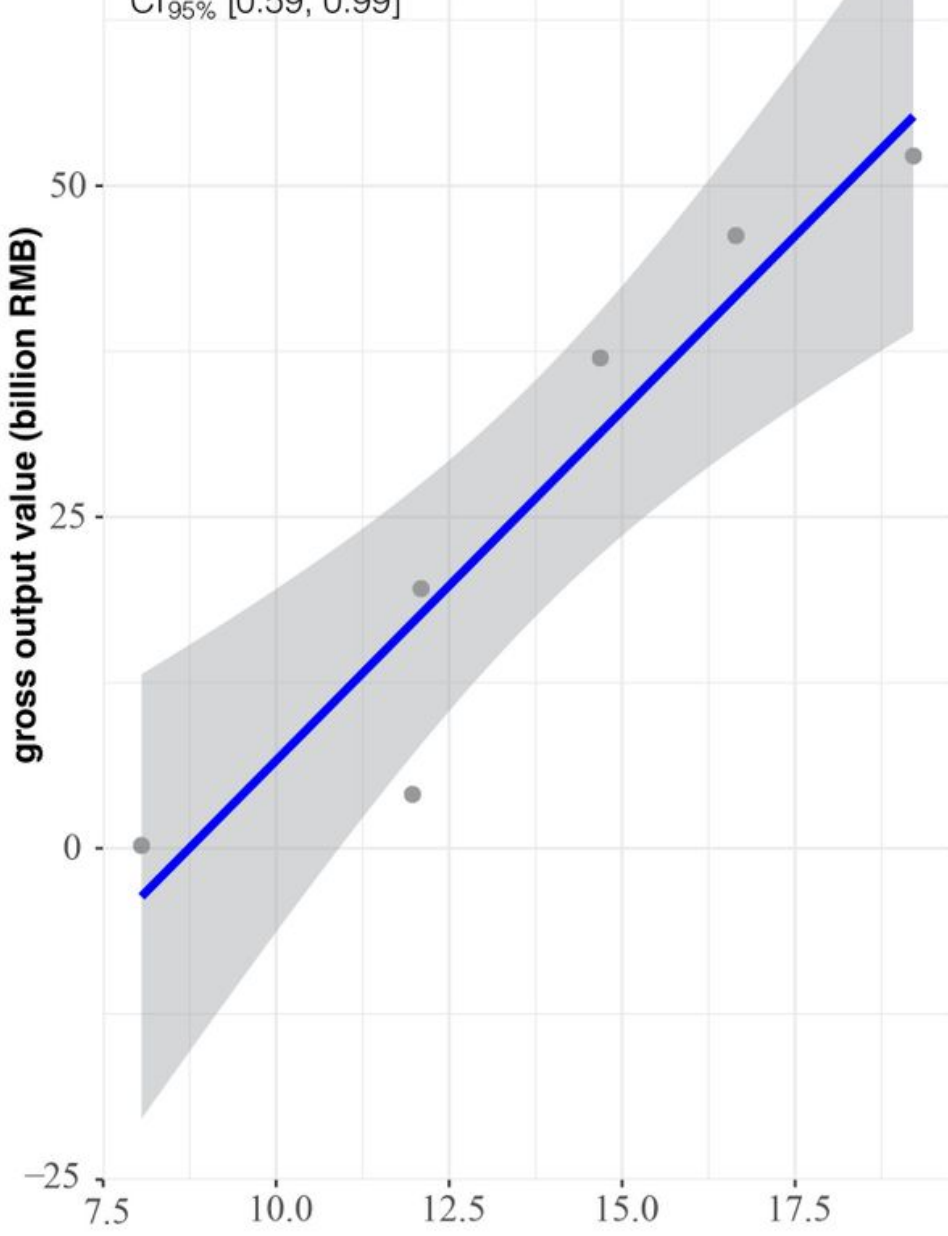

ARG counts $\mathrm{b}$

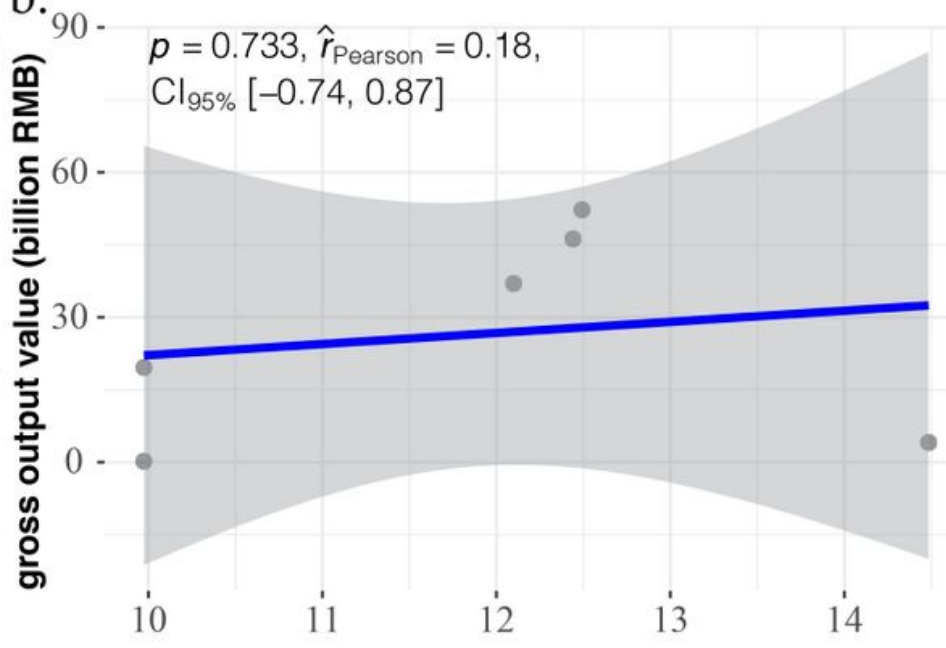

\section{plasmid replicon counts}

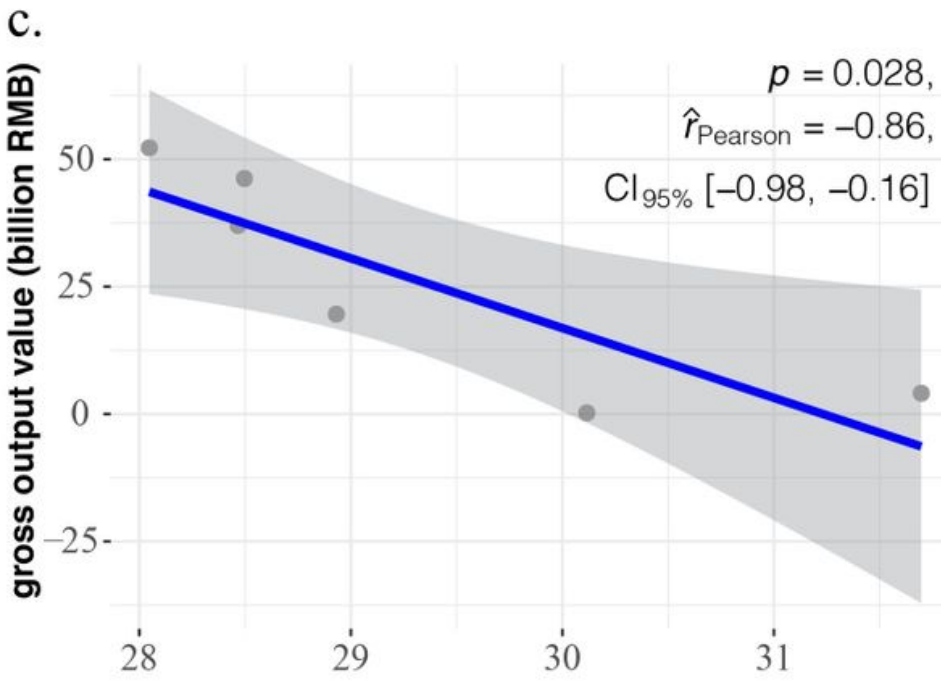

virulence gene counts

\section{Figure 4}

The correlation of antimicrobial production and resistance gene content. a. The correlation of gross output value of veterinary medicine and average count of ARGs detected from genomes. $b$. The correlation of gross output value of veterinary medicine and average count of plasmid replicons from genomes. c. The correlation of gross output value of veterinary medicine and average account of VGs detected from genomes. 


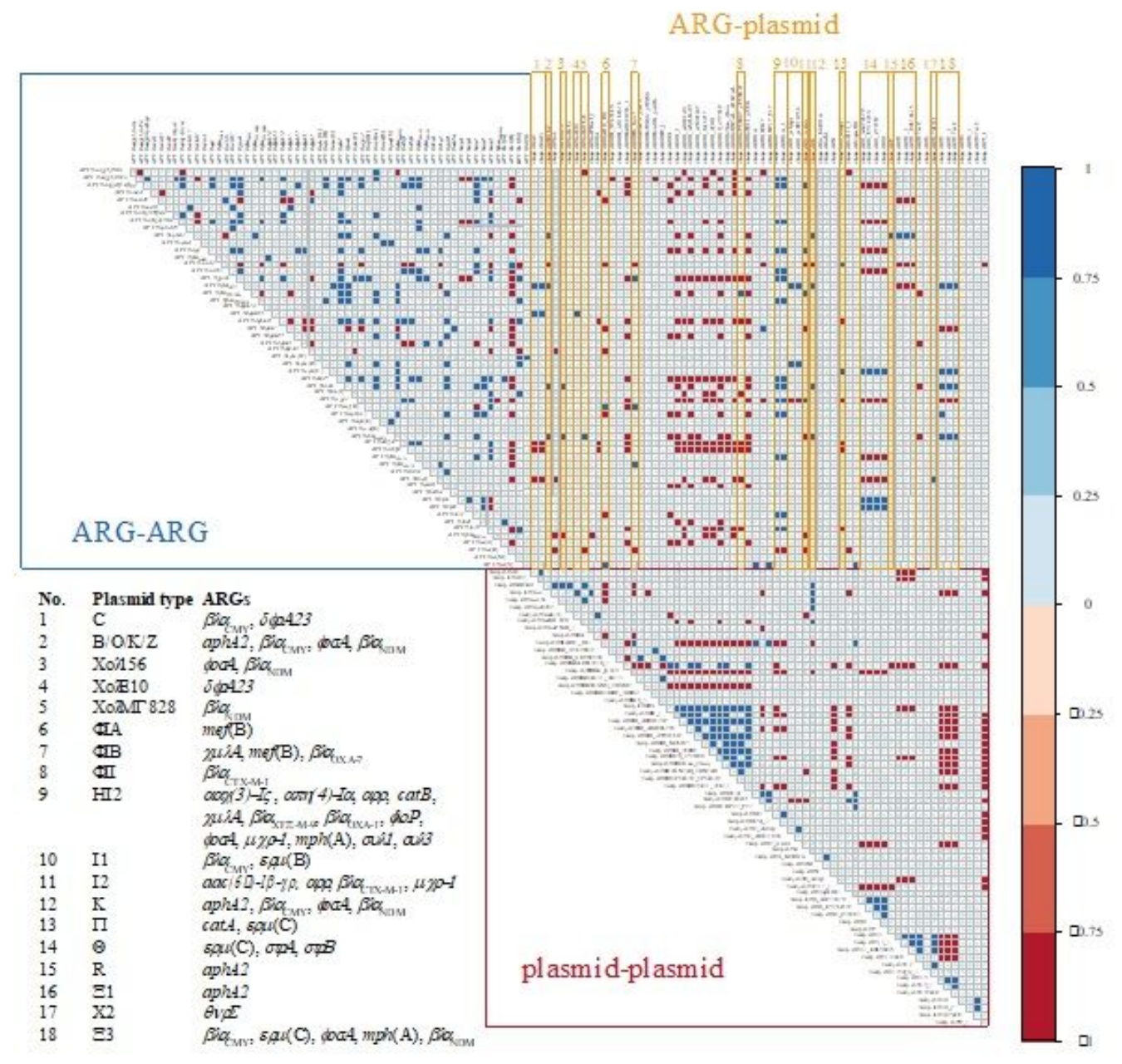

Figure 5

Co-occurrence of ARGs and plasmid replicons. We define the false discovery rate (FDR) threshold as 0.05 in this algorithm; significant pairwise values from 1 (blue) to -1 (red) (i.e. co-occurrence and mutual exclusivity) are shown. The blue box labelled "ARG-ARG" shows the pairwise co-occurrence and mutual exclusivity of ARGs, and the red box labelled "plasmidplasmid" shows the pairwise co-occurrence and mutual exclusivity of plasmid replicons. The gold boxes labelled with numbers indicate the co-occurrence and mutual exclusivity between ARGs and plasmid replicons, and these are annotated on the bottom left 
1970s-1990s $(\mathrm{n}=146)$
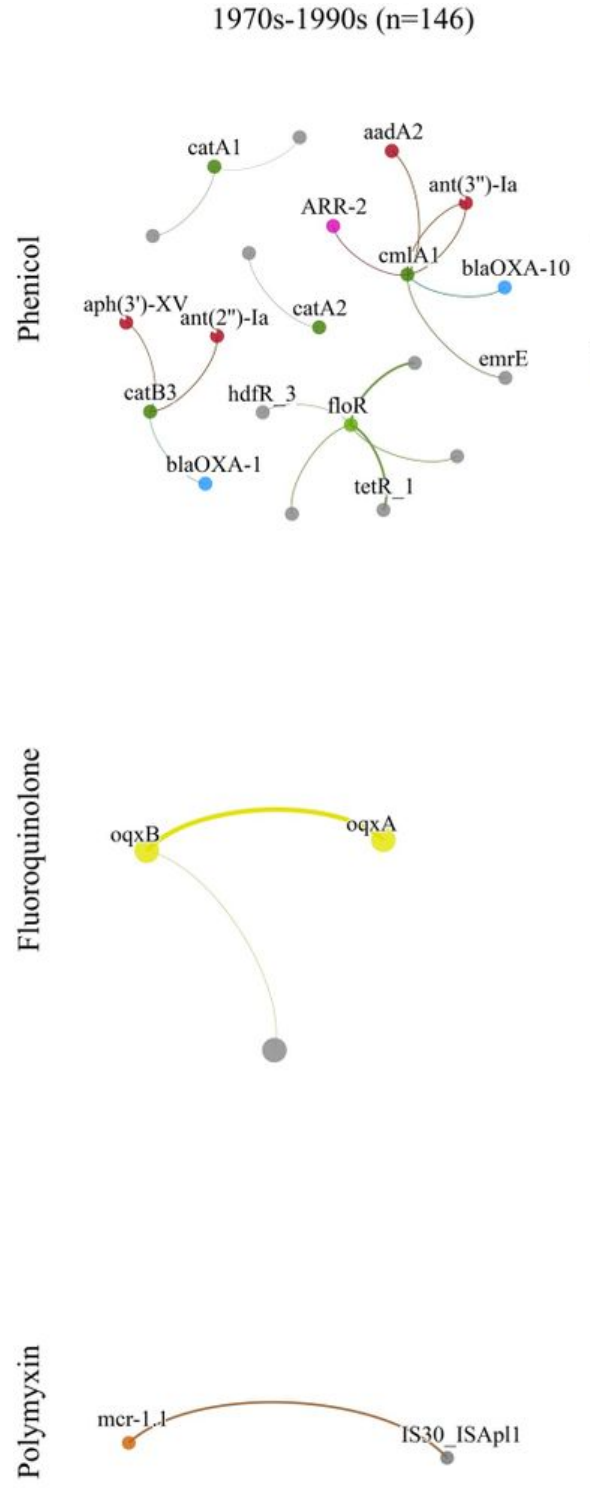

$2000 \mathrm{~s}(\mathrm{n}=169)$
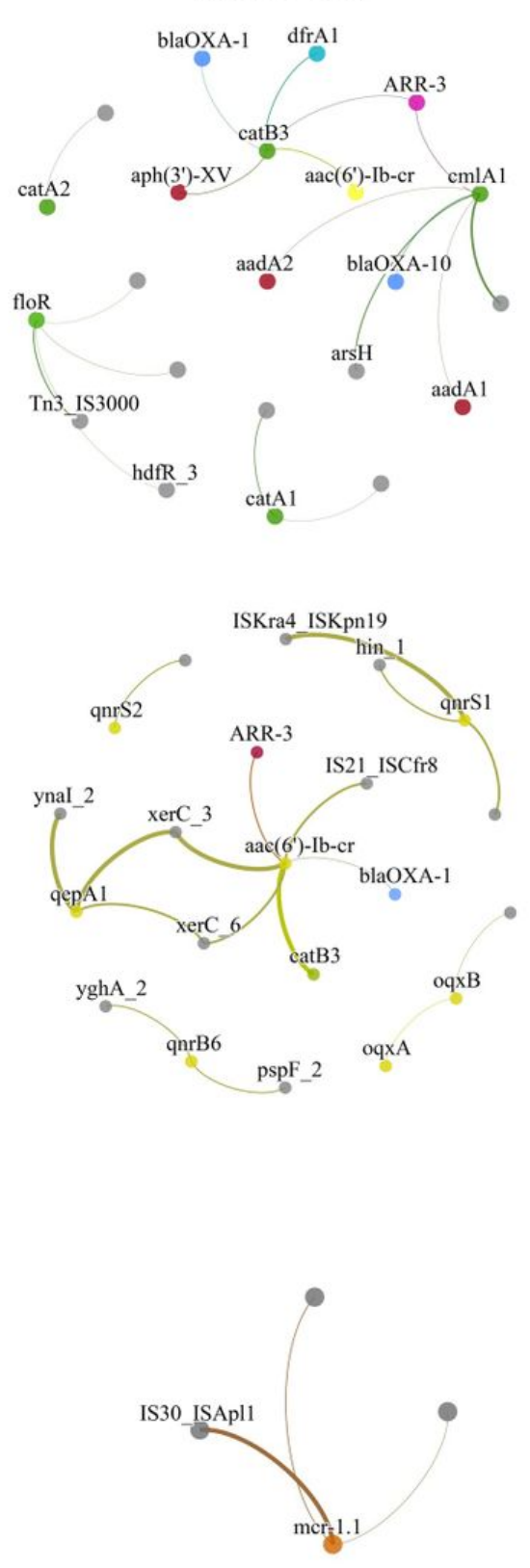

$2010 \mathrm{~s}(\mathrm{n}=667)$
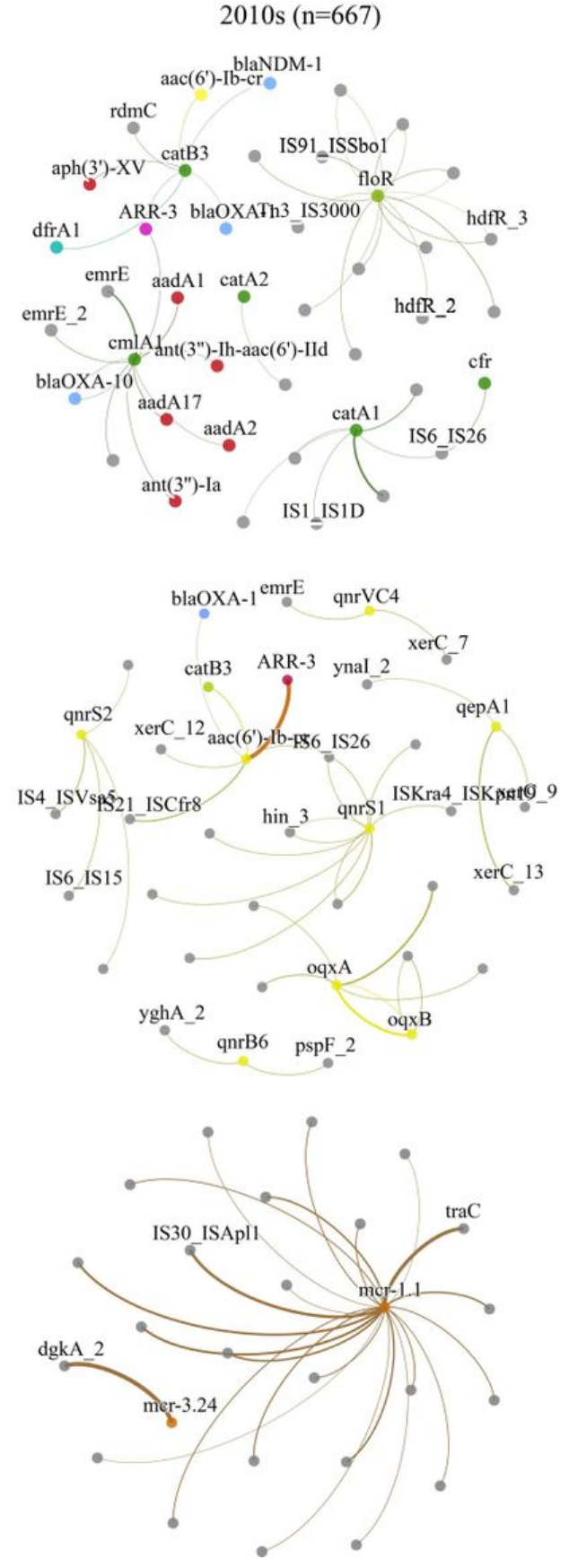

Figure 6

ARG co-occurrence networks of phenicol, fluoroquinolone and polymyxin resistance genes among isolates grouped by sampling time: 1970s-1990s ( $n=146), 2000 s(n=169)$ and 2010s $(n=667)$. Nodes represent genes and are colored to represent the antimicrobial class for ARGs; aminoglycoside: red; $\beta$-lactam: blue; trimethoprim: cyan; phenicol: green; rifampin: pink; fluroquinolones: yellow; poymyxins: orange; grey: non-ARG. Unlabelled nodes were annotated as hypothetical protein. Edges are coloured according to the nodes the connect. Edge widths represent connection weight calculated by Roary.

\section{Supplementary Files}

This is a list of supplementary files associated with this preprint. Click to download.

- TableS1S3.xlsx 
- EvolutionofAMRinanimalSupplementary.docx

Page 20/20 